\title{
BMJ Open What treatments work for anxiety in children with chronic fatigue syndrome/ myalgic encephalomyelitis (CFS/ME)? Systematic review
}

\author{
Sarah Victoria Ellen Stoll, ${ }^{1}$ Esther Crawley, ${ }^{1}$ Victoria Richards, ${ }^{1}$ Nishita Lal, ${ }^{1}$ \\ Amberly Brigden, ${ }^{1}$ Maria E Loades ${ }^{2}$
}

To cite: Stoll SVE, Crawley E, Richards V, et al. What treatments work for anxiety in children with chronic fatigue syndrome/myalgic encephalomyelitis (CFS/ME)? Systematic review. BMJ Open 2017;7:e015481. doi:10.1136/ bmjopen-2016-015481

- Prepublication history and additional material are available online. To view these files, please visit the journal online (http://dx.doi.org/10.1136/ bmjopen-2016-015481).

Received 9 December 2016 Revised 10 July 2017 Accepted 14 July 2017

\section{CrossMark}

${ }^{1}$ Population Health Sciences, Bristol Medical School, University of Bristol, Bristol ${ }^{2}$ Department of Psychology, University of Bath, Bath, UK

Correspondence to

Dr. Esther Crawley;

esther.crawley@bristol.ac.uk

\section{ABSTRACT}

Objectives Anxiety is more prevalent in children with chronic fatigue syndrome/myalgic encephalomyelitis (CFS/ $\mathrm{ME}$ ) than in the general population. A systematic review was carried out to identify which treatment methods are most effective for children with CFS and anxiety.

Design Systematic review using search terms entered into the Cochrane library and Ovid to search the databases Medline, Embase and psychINF0.

Participants Studies were selected if participants were $<18$ years old, diagnosed with CFS/ME (using US Centers for Disease Control and Prevention, the National Institute for Health and Care Excellence or Oxford criteria) and had a valid assessment of anxiety.

Interventions We included observational studies and randomised controlled trials.

Comparison Any or none.

Outcomes Change in anxiety diagnostic status and/ or change in anxiety severity on a validated measure of anxiety from pretreatment to post-treatment.

Results The review identified nine papers from eight studies that met the inclusion criteria. None of the studies specifically targeted anxiety but six studies tested an intervention and measured anxiety as a secondary outcome. Of these studies, four used a cognitive behavioural therapy (CBT)-type approach to treat CFS/ $\mathrm{ME}$, one used a behavioural approach and one compared a drug treatment, gammaglobulin with a placebo. Three of the CBT-type studies described an improvement in anxiety as did the trial of gammaglobulin. As none of the studies stratified outcomes according to anxiety diagnostic status or severity, we were unable to determine whether anxiety changed prognosis or whether treatments were equally effective in those with comorbid anxiety compared with those without.

Conclusion We do not know what treatment should be offered for children with both anxiety and CFS/ME. Further research is therefore required to answer this question.

Trial registration number This review was registered on Prospective Register of Systematic Review Protocols (PROSPERO) and the protocol is available from http:// www.crd.york.ac.uk/PROSPER0/display_record.asp?ID= CRD42016043488.

\section{Strengths and limitations of this study}

- This systematic review identified publications investigating the treatment of anxiety in children with chronic fatigue syndrome/myalgic encephalomyelitis

- Each article was screened and the data were extracted independently by two reviewers.

- Foreign articles were included and translators were recruited to assist where necessary.

- However, the grey literature was not searched.

- The findings of the review are limited by the exclusion of children with high levels of anxiety from some treatment trials.

\section{BACKGROUND}

Chronic fatigue syndrome (CFS)/myalgic encephalomyelitis (ME) is a chronic condition of unknown aetiology consisting of disabling fatigue, malaise, difficulty sleeping, joint/muscle aches and difficulty concentrating. ${ }^{1}$ The prevalence of CFS/ME in teenagers varies from $0.5 \%$ to $2.4 \%$, depending on the diagnostic criteria and methodology used. ${ }^{2-4} \mathrm{CFS} / \mathrm{ME}$ can have a very debilitating impact on children with one study showing $\mathrm{a} \leq 40 \%$ school attendance rate in almost two-thirds $(62 \%)$ of children with CFS $/ \mathrm{ME}^{5}$ Furthermore, children with CFS/ME experience difficulty concentrating and impairments in cognitive function which have a significant impact on their learning and education. ${ }^{6}$

Anxiety is a relatively common mental health condition; in the general population, it is estimated that $5 \%-19 \%$ of all children suffer from anxiety. ${ }^{7}$ Children with CFS/ME experience higher rates of anxiety than the normal population, with one study showing rates of $38 \%$ in teenage girls. ${ }^{8}$ Specifically, separation anxiety and social phobia were found to be the most prevalent subtypes 
of anxiety in paediatric CFS/ME. ${ }^{8}$ Children with a chronic illness might be more anxious as a reaction to being ill, the 'threatening environment' of a chronic illness or other psychological factors as a result of their condition. ${ }^{9}$

It is unclear as to whether children with CFS/ME develop anxiety as a result of their condition or whether psychological difficulties might pose a vulnerability to developing $\mathrm{CFS} / \mathrm{ME}^{2}$ or whether an external factor might increase the likelihood of an individual developing both anxiety and CFS/ME. Being diagnosed with CFS/ ME has a substantial impact on social and academic life, which could potentially contribute to the development of distress, including depression and/or anxiety. ${ }^{10}$ This may be compounded by the stigma surrounding CFS/ME and the inability to fully explain this illness, resulting in uncertainty. ${ }^{10}{ }^{11}$ It is also possible that a biological mechanism is responsible for both the development of CFS/ME and anxiety, with some evidence of cortisol levels being implicated in CFS/ME in children and clear evidence of cortisol being linked to anxiety. ${ }^{12-14}$

Anxiety may have a negative impact on recovery in paediatric CFS/ME by affecting an individual's ability to follow the evidence-based treatment for CFS/ME, which includes gradually increasing their activity levels. For example, in children, if the aim of treatment for CFS/ ME was to gradually increase school attendance; anxiety about going to school may prevent them from doing this. Therefore, comorbid anxiety may need to be a specific treatment target in paediatric CFS/ME. The aims of this review are to establish what is known about treatment approaches for anxiety in children with CFS/ME and what is known about the impact of comorbid anxiety on outcome in CFS/ME.

\section{METHODS}

\section{Data sources and search strategy}

The search strategy for this systematic review incorporated the use of the Cochrane library and OVID to search the databases Medline, Embase and psychINFO. The search strategy was designed with input from an information specialist to include the concepts 'paediatric' and 'CFS/ME'. Limits were applied according to the inclusion criteria. Final searches were conducted in July 2016. The full protocol can be found in Prospective Register of Systematic Review Protocols (PROSPERO) (http:// www.crd.york.ac.uk/PROSPERO/display_record.asp? ID=CRD42016043488). The protocol was not revised after registration, although the search terms were slightly amended, and the final search terms are available as online supplementary information 1.

The grey literature was not searched, but the reference lists of all the included articles were hand searched. Foreign studies were considered for inclusion with the help of native speakers who assisted in the translation of these to determine whether the studies met the inclusion criteria.
Inclusion and exclusion criteria

Studies were included if they met the following criteria:

Participants

- Children $<18$ years of age;

- Diagnosed with CFS/ME defined using US Centers for Disease Control and Prevention (CDC) criteria $^{15}$ or the National Institute for Health and Care Excellence (2007) or Oxford criteria.

\section{Interventions}

- Longitudinal study (treatment trial or observational cohort study).

\section{Comparison}

- Any or none.

\section{Outcomes}

- Study included a validated assessment of anxiety at baseline and repeated measures for either anxiety or fatigue on a validated scale.

\section{Study selection}

Initial screening was by title and abstract to assess eligibility. Subsequently, full texts of the potentially eligible articles were reviewed to ascertain whether they met all the eligibility criteria. Two reviewers (from a pool of five reviewers, including SS, ML, VR, NL and $\mathrm{AB}$ ) independently assessed papers at each stage. Differences in opinion were resolved by discussion, overseen by EC, with reference to the review protocol.

\section{Data extraction}

For all included articles, data were extracted using a data extraction form, collecting information such as the CFS/ ME definition used, treatment/interventions provided, definition of response, details of the setting of the study, how children were recruited for the study, date of the study and child characteristics (including age). Three reviewers (SS, ML, NL) independently carried out this process.

\section{Quality assessment}

Two reviewers independently assessed the quality of the studies included using the Cochrane risk of bias assessment tool as well as Critical Appraisal Skills Programme tools. ${ }^{16-18}$ This was done for both observational studies and randomised controlled trials (RCTs).

\section{Data synthesis}

There was insufficient comparable data to undertake a meta-analysis. Therefore, a narrative synthesis was undertaken.

\section{RESULTS}

Identification of studies

A total of 1274 records were found by database searching, and after duplicates were removed, 1074 remained 


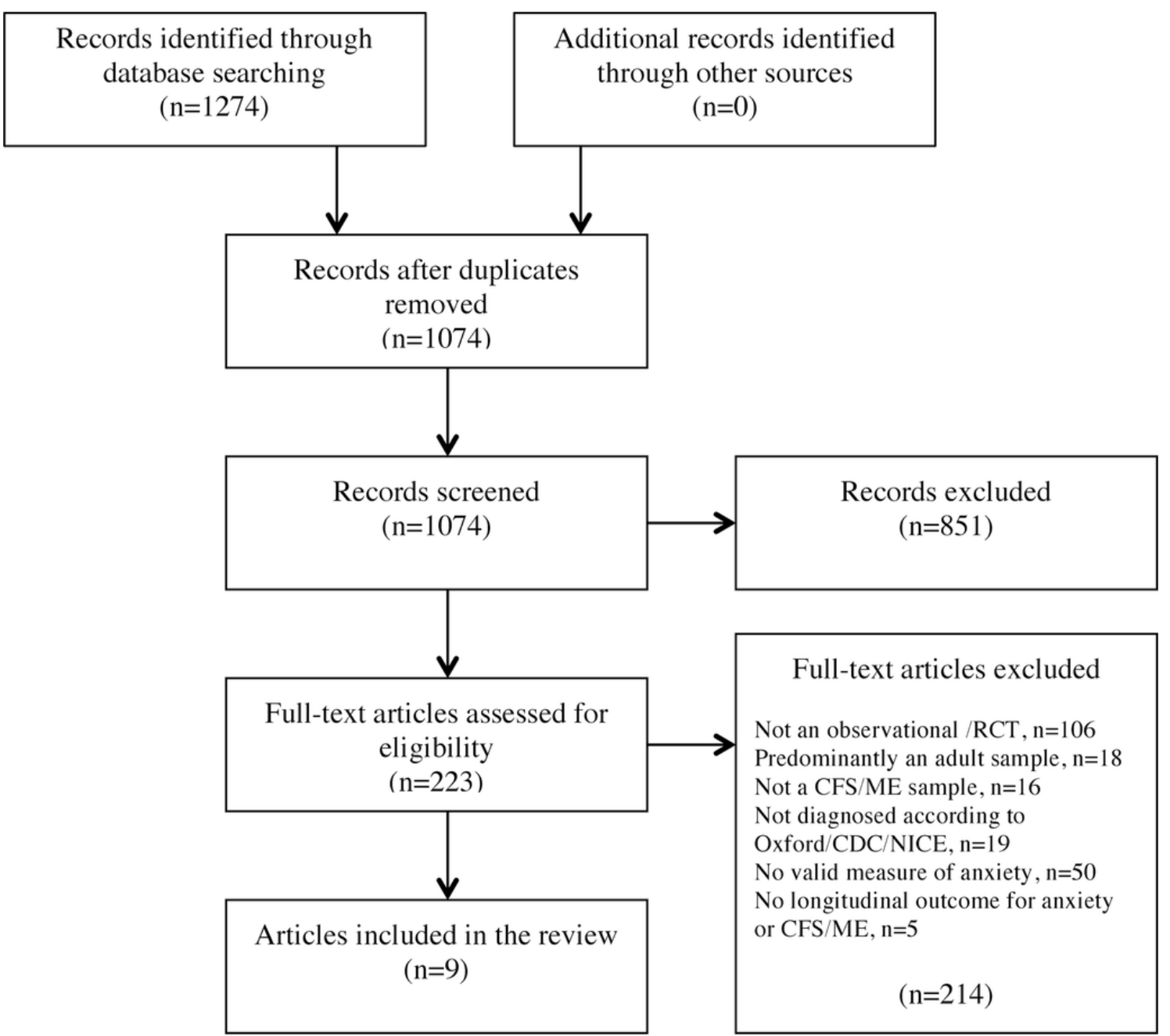

Figure 1 Systematic review flow chart (based on PRISMA guidelines). ${ }^{40}$ CDC, US Centers for Disease Control and Prevention; CFS, chronic fatigue syndrome; ME, myalgic encephalomyelitis; NICE, the National Institute for Health and Care Excellence; PRISMA, Preferred Reporting Items for Systematic Reviews and Meta-Analyses; RCT, randomised controlled trials.

(figure 1). Two hundred and twenty-three articles were reviewed in full; however, only nine papers were eligible from eight studies. One foreign paper (Spanish) was included in this review, and a further three were considered ineligible at full-text review (Dutch, German and Spanish).

\section{Quality assessment}

There were five observational studies and three RCTs that were included in this systematic review. Most of the studies had a clear focused aim, although none of the included studies specifically focused on change in anxiety in paediatric CFS/ME as their primary outcome. The participants were recruited in an acceptable way for most studies. The RCTs were judged most robust and least at risk of bias; the participant groups were comparable at the point of randomisation and the groups were also treated equally apart from the experimental treatment under investigation. One RCT used a placebo, ${ }^{19}$ while the other two used an active treatment comparison (treatment as usual). ${ }^{620-22}$ For the observational studies, one was a case study, and therefore at high risk of bias. ${ }^{23}$ For the remainder of the observational studies, ${ }^{24-26}$ risk of bias was either low or unclear; exposure and outcome were measured accurately to reduce bias in most studies and follow-up deemed long enough. The quality assessment is available as supplementary information in online supplementary information 2.

\section{Patient and study characteristics}

Anxiety was measured using self-report questionnaires including the Hospital Anxiety and Depression Scale (HADS) ${ }^{27}$ the State-Trait Anxiety Inventory for Children (STAIC), ${ }^{28}$ Spence Children's Anxiety Scale $(\mathrm{SCAS})^{29}$ and the Multidimensional Anxiety Scale for Children (MASC).$^{30}$ One study used a diagnostic interview, the Development and Well-Being Assessment (DAWBA) ${ }^{31}$ (see table 1).

Sample sizes ranged from 1 to 135 (see table 1) and ages ranged from 11 to 19 years. Most studies diagnosed participants according to the CDC criteria. ${ }^{15}$ The majority of participants were female in all of the studies, which is consistent with the epidemiology of adolescent CFS/ ME. ${ }^{32}$ 


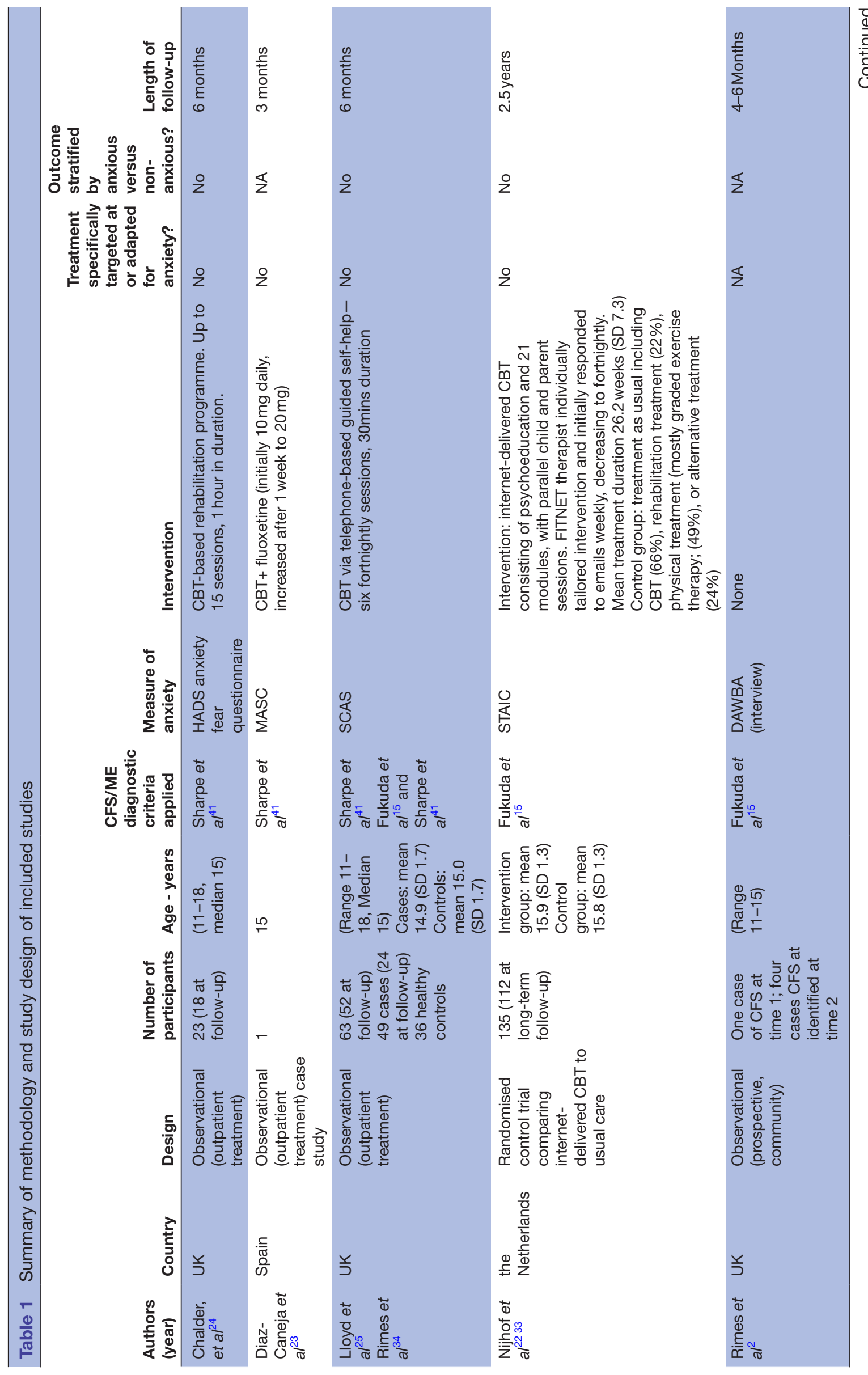




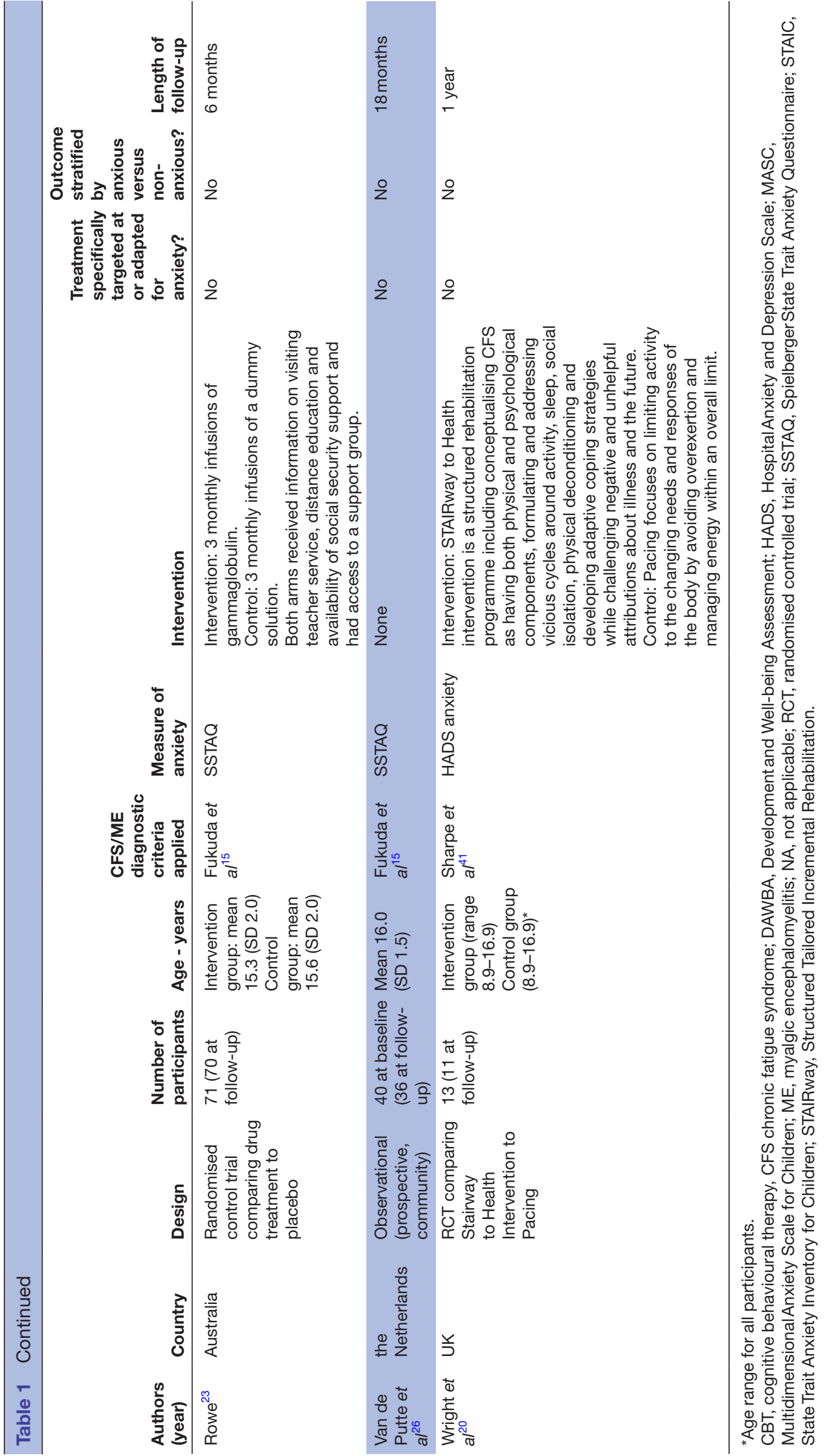


Table 2 Details of components in provided in CBT and behavioural interventions

\begin{tabular}{|c|c|c|}
\hline Study & Intervention & $\begin{array}{l}\text { Duration and } \\
\text { frequency }\end{array}$ \\
\hline $\begin{array}{l}\text { Chalder et } \\
a l^{24}\end{array}$ & $\begin{array}{l}\text { CBT-based rehabilitation programme including graded approach to increasing activity } \\
\text { and establishing a sleep routine. Cognitive work was included where necessary. }\end{array}$ & $\begin{array}{l}\text { Up to } 15 \text { hourly } \\
\text { sessions, face to face }\end{array}$ \\
\hline $\begin{array}{l}\text { Diaz-Caneja } \\
\text { et } a l^{23}\end{array}$ & $\begin{array}{l}\text { CBT (no further details given)+fluoxetine (initially } 10 \mathrm{mg} \text { daily, increased after } 1 \text { week to } \\
20 \mathrm{mg} \text { ). }\end{array}$ & No details given. \\
\hline $\begin{array}{l}\text { Lloyd et } a l^{25} \\
\text { Rimes et } a l^{34}\end{array}$ & $\begin{array}{l}\text { CBT which addressed unhelpful beliefs including fears about symptoms/activity. Activity } \\
\text { diaries were used to establish a consistent routine and achieve a balance between } \\
\text { activity and rest. The programme emphasised gradually increasing activities, including } \\
\text { school, home, socialising and exercise and establishing a regular sleep routine. Social } \\
\text { and emotional problems addressed if time allowed. }\end{array}$ & $\begin{array}{l}\text { Up to } 6 \times 30 \text { min } \\
\text { sessions, by } \\
\text { telephone, based on } \\
\text { self-help manual }\end{array}$ \\
\hline $\begin{array}{l}\text { Nijhof et } \\
a l^{22} 33\end{array}$ & $\begin{array}{l}\text { CBT in the FITNET programme consisted of two sections, a psychoeducational section } \\
\text { and CBT section. Parents had parallel modules. }\end{array}$ & $\begin{array}{l}21 \text { interactive } \\
\text { modules delivered } \\
\text { via the internet, with } \\
\text { e-consultations from } \\
\text { therapists. }\end{array}$ \\
\hline
\end{tabular}

CBT, cognitive behavioural therapy; CFS, chronic fatigue syndrome; STAIRway, Structured Tailored Incremental Rehabilitation; FITNET , Fatigue In Teenagers on the interNET.

\section{Treatment in anxiety for children with CFS/ME}

No studies specifically targeted anxiety in children with CFS/ME. Of the eight studies included, two ${ }^{226}$ were longitudinal observational cohort studies. These two studies did not test an intervention and were therefore uninformative for establishing what is known about treatments for children with CFS/ME and anxiety.

Of the six treatment studies, four used a CBT approach, one used a behavioural approach and one used intravenous gammaglobulin. The primary outcomes included fatigue $^{2022} 24253$, disability or function ${ }^{23}$ and school attendance. ${ }^{2022} 24253334$ All studies measured anxiety as a secondary outcome.

The common elements of all five cognitive behavioural and behavioural interventions appear to be the inclusion of a graded approach to managing activity and employing strategies to address cognitive elements such as illness-related beliefs and negative predictions about the future where necessary (see table 2 for details). Interventions varied considerably in the duration of treatment (12 weeks to 1 year), length of sessions (no direct therapist contact/30 min/60 min) and treatment modality (faceto-face, telephone, internet-delivered modules with therapist e-consults).

\section{Studies using a CBT approach}

The duration of CBT across the studies ranged from 6 to 30 min telephone sessions at fortnightly intervals ${ }^{25} 34$ to 21 internet session modules over 26 weeks. ${ }^{22}{ }^{33}$ In three of the four studies, the authors report that anxiety improved with treatment, which suggests that cognitive behavioural treatment for CFS/ME may improve anxiety (table 3).

Nijhof et als RCT compared internet-based CBT with traditional methods in 135 participants. The internet-based CBT, FITNET, includes psycho-educational modules for patients and parents in addition to CBT modules developed by the Expert Centre for Chronic Fatigue. ${ }^{22}{ }^{33}$ Patients were able to send emails and therapists replied to 'e-consults' on the same day each week or according to the treatment plan. At 6 months post-randomisation, the study demonstrated a significant improvement in school attendance (full-time school $75 \%$ in FITNET group compared with $16 \%$ in usual care group), fatigue and physical function in those receiving the FITNET intervention with $63 \%$ defined as 'recovered' compared with $8 \%$ of those receiving treatment as usual.

In the treatment study by Chalder et al, 23 participants were offered family-based CBT. There was a significant improvement in anxiety (measured using the HADS) at 6 months (Median (IQR) $7(6.7,9.7)$ at assessment to $0.5(0.5,9) .{ }^{24}$ The family-based CBT involved 15 fortnightly hourly sessions using a graded therapy method including a sleep routine and was implemented by patients and family with therapist guidance. The goal in this study was for children to return to full-time education. Activity goals were set to include tasks such as walking, school work and attending social events. The activities were slowly increased and the aim to disassociating symptom relief with activity cessation. A sleep 


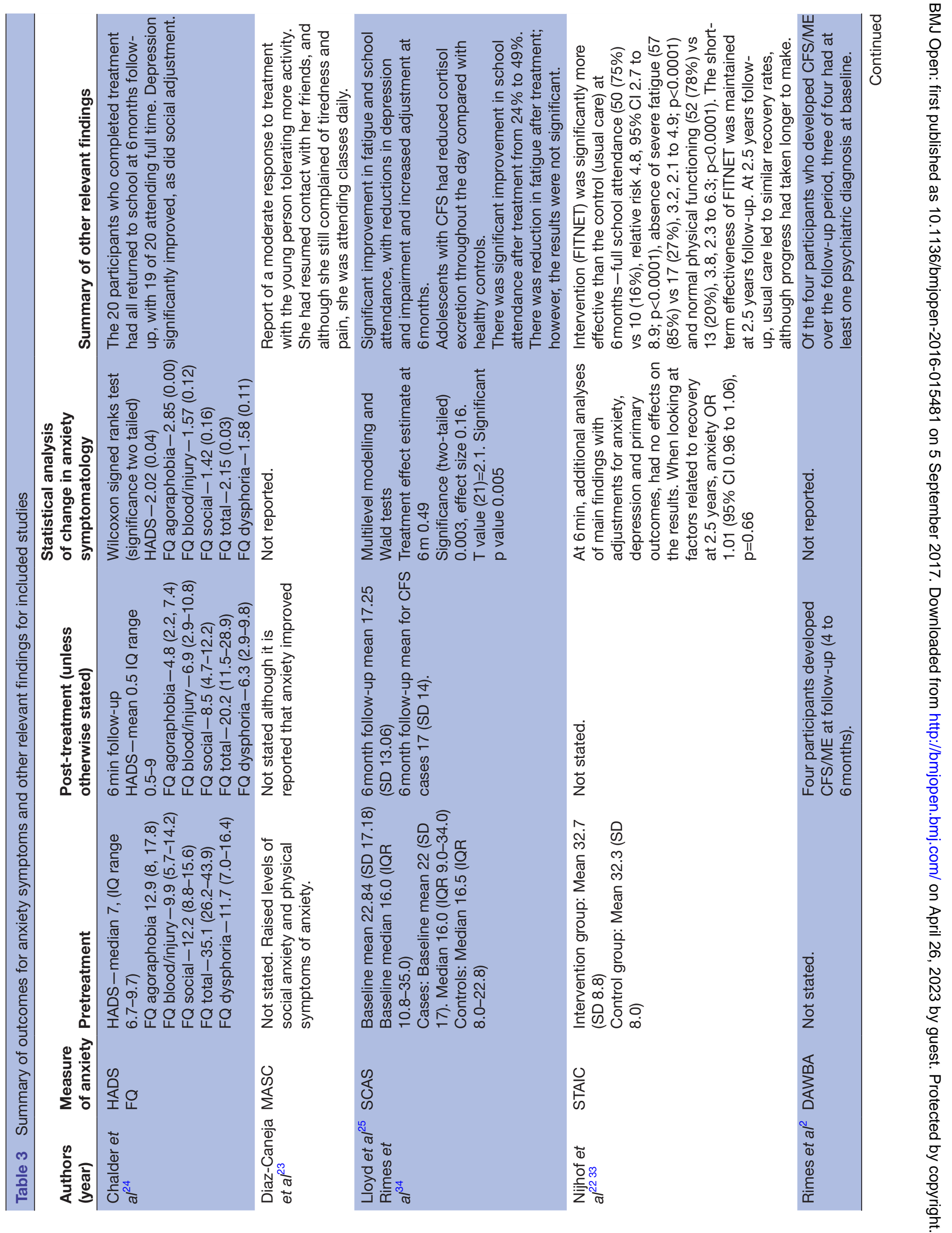




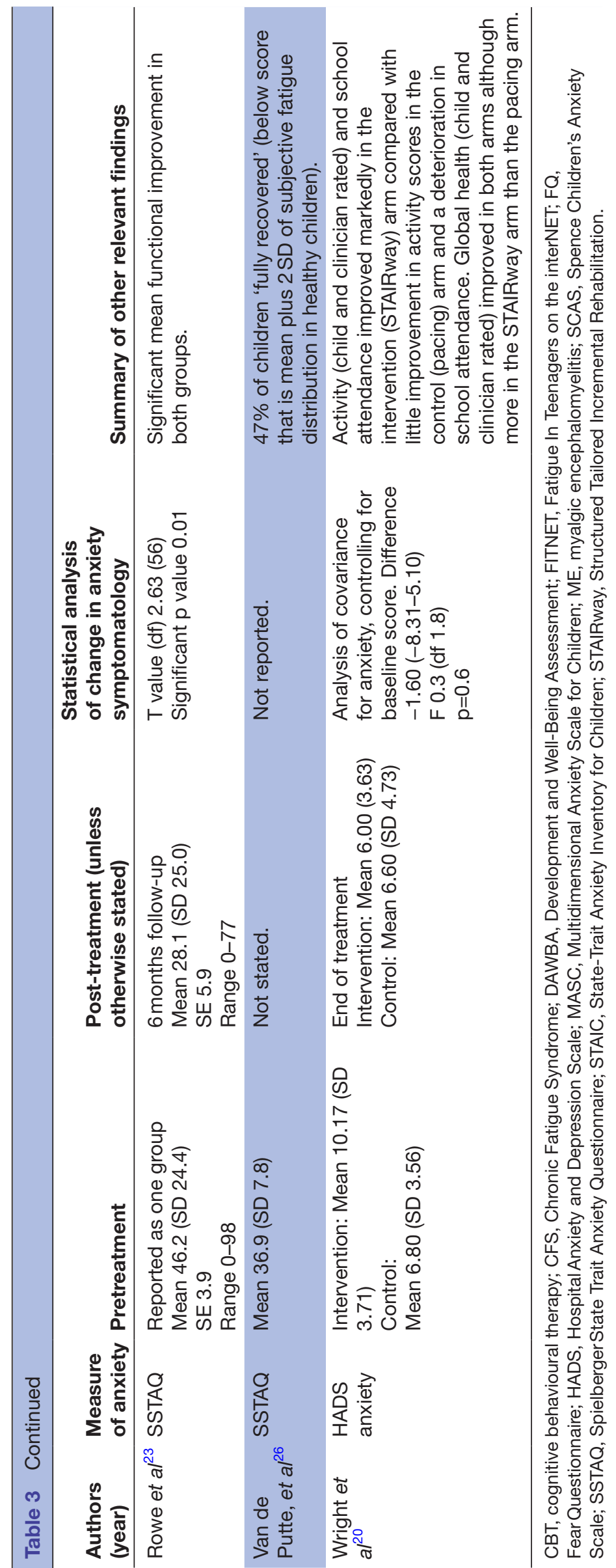


routine was also established in addition to changing perceptions of their illness to prevent negative thoughts.

Lloyd et al trialled a telephone self-help intervention involving 63 participants undergoing 6 fortnightly 30 min sessions based on a CBT model that also showed a significant improvement in anxiety levels (treatment effect estimate -0.49 (CI -0.82 to 0.17 ), $\mathrm{p}=0.003$ ). ${ }^{25}$ This approach addressed any fears the participants had towards the programme in addition to completing activity diaries and developing a better sleep routine. Fatigue and school attendance were the primary outcomes, with anxiety being a secondary outcome measure.

Diaz-Caneja et al reported a moderate response to combined CBT and fluoxetine treatment in a single case study $(n=1) .{ }^{23}$ They found that with this approach, there was increased tolerance to activity although the subject still felt tired. However, the specific components of treatment are not clear and the treatment appears to have been ongoing at the time of writing the case study.

\section{Study using a behavioural approach}

Wright et al's study was an RCT comparing two behavioural approaches, one called 'pacing' and the other 'STAIRway to health'. ${ }^{20}$ Thirteen children were randomised into the treatment groups with stratification for age, sex and mobility. The 'pacing' arm involved exercising to the child's limits while adapting to an individual's bodily needs. The 'STAIRway to health' arm was a structured tailored incremental rehabilitation programme that took a more holistic approach to CFS/ ME aiming to treat both physical and psychological symptoms including nutrition, sleep, social activities and emotional issues. ${ }^{20}$ The clinic appointments were weekly for 1 month, fortnightly for the next 3 months, every third week for 2 months and every 4 weeks for 6 months. STAIRway had a greater emphasis on coping strategies to deal with both the physical and psychological implications of CFS/ME and showed a greater improvement in anxiety levels. ${ }^{20}$

\section{Study using a pharmacological treatment}

In the study by Rowe, 71 patients were recruited into an RCT comparing intravenous gammaglobulin to a placebo. ${ }^{23}$ Four domains were investigated, including school attendance, amount of school work attempted, amount of physical activities attempted and amount of social activities attempted. Anxiety reduced in all participants at 6 months follow-up, both in those who were treated with the medication intravenous gammaglobulin and in those who received a placebo. ${ }^{23}$

\section{Outcome for children with CFS/ME in those who are anxious versus those who are not}

Neither the longitudinal observational cohort studies nor the treatment studies that assessed the outcome for children with CFS/ME who are anxious compared with those who were not. Some studies excluded those who were above a significant threshold for anxiety (Nijhof $e t$ $\left.a l^{33}\right)$ as shown in table 3 .

\section{Variation of outcome in children with CFS/ME and comorbid anxiety}

None of the studies compared the outcome between those with and without anxiety.

\section{DISCUSSION}

This is the first systematic review to investigate the outcomes and treatment of children with CFS/ME who are also anxious. From this review, we know that treatment using a cognitive behavioural or behavioural approach led to improvements in self-reported anxiety at follow-up. However, the existing research is limited by the small sample sizes which are not powered to detect a treatment effect in the treatment of anxiety, inconsistency in the measurement of anxiety and the exclusion of patients with high levels of anxiety from some treatment trials. ${ }^{23}$

The strengths of this review include a thorough and wide-ranging search strategy by using a number of databases in addition to hand-searching articles. Five reviewers carried out screening, with at least two reviewers screening at each stage. An additional reviewer was consulted to resolve differences of opinion. Foreign language papers were included with the help of native speakers to aid in translation.

Only eight studies were found with most having small sample sizes. None were powered to determine treatment efficacy in those with CFS/ME and anxiety. Only three of the studies were RCTs and one excluded those with high anxiety scores, ${ }^{19} 202233$ making it difficult to investigate treatment effects in those with comorbid anxiety. None of the studies included children who were 10 years old and younger and therefore we do not know about treatment efficacy in this group. ${ }^{33}$

It is difficult to determine from the results of these studies whether anxiety scores have improved due to regression to the mean, anxiety reducing on its own without intervention or whether the treatment itself is having an effect. Improvements in functioning may lead to increased exposure to anxiety provoking situations (for example, school), resulting in a habituation response. Therefore, it is not possible to ascertain the extent to which treatment was responsible for improvements, given the lack of robust studies, designed to specifically compare treatment for anxiety in paediatric CFS/ME patients to waiting list controls (or an alternative treatment/usual care).

The improvements in anxiety reported in the study by Rowe in both the pharmacological treatment arm and the placebo arm suggests that anxiety in CFS/ME may naturally decrease over time without active intervention. ${ }^{23}$ This finding may be explained by the mean functional improvement that demonstrated a significant reduction in both groups; that is, anxiety might improve as a result of functionally improving. However, this is difficult 
to disentangle as both groups received information on education and social support services in this study, and this in itself may have been an active intervention that led to changes in functioning and anxiety. ${ }^{23}$

For children without comorbid physical health conditions who present for treatment of anxiety, various interventions have shown to be effective, including CBT, bibliotherapy (parents given a type of instruction manual to aid their children's' anxiety) and e-therapies (computerised programme).$^{35}$ However, whether these therapies will be effective in paediatric CFS/ME is uncertain. As rates of anxiety are increased in children with CFS/ME, by remediating their fatigue, anxiety may decrease. ${ }^{8}$

This review did not identify any studies that clarify the impact of anxiety on outcome in CFS/ME (with or without treatment). In adults with CFS/ME, one study has found that anxiety improved in CFS/ME patients receiving CBT, graded exercise therapy and activity management. ${ }^{36}$ In other childhood chronic illnesses such as inflammatory bowel disease, CBT techniques have shown to be beneficial. ${ }^{37} \mathrm{CBT}$ has also been found to be effective for children with type 1 diabetes. ${ }^{38}$ A systematic review concluded that despite weak evidence, CBT is beneficial in children with chronic physical illness and comorbid anxiety. ${ }^{39} \mathrm{On}$ this basis, and as CBT has been found to be successful for anxiety in children in the general population, this does seem like the most promising approach. Further research to determine the impact of anxiety on recovery, and if necessary, to adapt CBT for CFS/ME to include anxiety management components, would be beneficial.

\section{CONCLUSION}

Paediatric CFS/ME is a severe debilitating illness causing significant levels of school absence. About a third of children with CFS/ME have high levels of anxiety. We wanted to find out what was known about treatment approaches for anxiety in children with CFS/ME and what is known about the impact of comorbid anxiety on outcome in $\mathrm{CFS} / \mathrm{ME}$. While CBT appears to result in lower levels of anxiety at follow-up, there was insufficient evidence to conclude what the best treatment is for dealing with anxiety in paediatric CFS/ME patients.

Acknowledgements The authors would like to thank Catherine Borwick and Roxanne Parslow for their assistance in developing the search strategy. The authors also very much appreciate the time taken by colleagues who are native foreign language speakers who assisted with the translation.

Contributors $\mathrm{ML}$ conceived the study and designed the study protocol. SS, ML, NL, VR and $A B$ did the data search and data synthesis. EC resolved conflicts. SS drafted the manuscript and $\mathrm{ML}$ and $\mathrm{EC}$ reviewed and edited the manuscript. All authors reviewed the paper before submission.

Funding EC was funded by the NIHR (Senior Research Fellowship, SRFA2013A06A013). Dr Loades is funded by the NIHR (Doctoral Research Fellowship, DRFA2016A09A021). This report is independent research. The views expressed in this publication are those of the authors(s) and not necessarily those of the NHS, The National Institute for Health Research or the Department of Health.

Competing interests EC is a medical advisor for the Sussex \& Kent ME/CFS Society. There are no other conflicts of interest.

Provenance and peer review Not commissioned; externally peer reviewed.
Data sharing statement No additional data available. This systematic review is based on published research only. There was no new data collected in this research.

Open Access This is an Open Access article distributed in accordance with the Creative Commons Attribution Non Commercial (CC BY-NC 4.0) license, which permits others to distribute, remix, adapt, build upon this work non-commercially, and license their derivative works on different terms, provided the original work is properly cited and the use is non-commercial. See: http://creativecommons.org/ licenses/by-nc/4.0/

(c) Article author(s) (or their employer(s) unless otherwise stated in the text of the article) 2017. All rights reserved. No commercial use is permitted unless otherwise expressly granted.

\section{REFERENCES}

1. National Institute for Health and Clinical Excellence. Chronic fatigue syndrome/myalgic encephalomyelitis (or encephalopathy): diagnosis and management. Nice guideline 2007 https://www.nice.org.uk/ guidance/CG53/chapter/introduction.

2. Rimes KA, Goodman R, Hotopf M, et al. Incidence, prognosis, and risk factors for fatigue and chronic fatigue syndrome in adolescents: a prospective community study. Pediatrics 2007;119:e603-9.

3. Crawley E, Hughes R, Northstone K, et al; Chronic disabling fatigue at age 13 and association with family adversity. , 2012:130, e71.

4. Farmer A, Fowler T, Scourfield J, et al. Prevalence of chronic disabling fatigue in children and adolescents. Br J Psychiatry 2004:184:477-81.

5. Crawley E, Sterne JA. Association between school absence and physical function in paediatric chronic fatigue syndrome/myalgic encephalopathy. Arch Dis Child 2009;94:752-6.

6. Nijhof LN, Nijhof SL, Bleijenberg G, et al. The impact of chronic fatigue syndrome on cognitive functioning in adolescents. Eur $\mathrm{J}$ Pediatr 2016;175:245-52.

7. Costello E, Egger H, Angold A. Phobic and anxiety disorders in children and adolescents: a clinician's guide to effective psychosocial and pharmacological interventions. In: Ollendick TH, March JS, eds. Developmental epidemiology of anxiety disorders. New York: oxford University Press, 2004:61-91.

8. Crawley E, Hunt L, Stallard P. Anxiety in children with CFS/ME. Eur Child Adolesc Psychiatry 2009;18:683-9.

9. Pao M, Bosk A. Anxiety in medically ill children/adolescents. Depress Anxiety 2011;28:40-9.

10. Bould H, Lewis G, Emond A, et al. Depression and anxiety in children with CFS/ME: cause or effect? Arch Dis Child 2011;96:211-4.

11. Fisher H, Crawley E. Why do young people with CFS/ME feel anxious? A qualitative study. Clin Child Psychol Psychiatry 2013;18:556-73.

12. Nijhof SL, Rutten JM, Uiterwaal CS, et al. The role of hypocortisolism in chronic fatigue syndrome. Psychoneuroendocrinology 2014;42:199-206.

13. Greaves-Lord K, Ferdinand RF, Oldehinkel AJ, et al. Higher cortisol awakening response in young adolescents with persistent anxiety problems. Acta Psychiatr Scand 2007;116:137-44.

14. Schiefelbein VL, Susman EJ. Cortisol levels and longitudinal cortisol Change as Predictors of anxiety in adolescents. J Early Adolesc 2006;26:397-413.

15. Fukuda K, Straus S, Hickie I, et al. The chronic fatigue syndrome: a comprehensive approach to its definition and study. Ann Intern Med 1994;121:953-9.

16. Higgins JPT GS. Cochrane Handbook for Systematic Reviews of Interventions: Wiley Online Library, 2011.

17. Critical Appraisal Skills Programme. 11 questions to help you make sense of a trial, 2013.

18. Critical Appraisal Skills Programme. 12 questions to help you make sense of cohort study, 2013.

19. Rowe KS. Double-blind randomized controlled trial to assess the efficacy of intravenous gammaglobulin for the management of chronic fatigue syndrome in adolescents. J Psychiatr Res 1997;31:133-47.

20. Wright B, Ashby B, Beverley D, et al. A feasibility study comparing two treatment approaches for chronic fatigue syndrome in adolescents. Arch Dis Child 2005;90:369-72.

21. Nijhof SL, Bleijenberg G, Uiterwaal CS, et al. Effectiveness of internet-based cognitive behavioural treatment for adolescents with chronic fatigue syndrome (FITNET): a randomised controlled trial. Lancet 2012;379:1412-8. 
22. Nijhof SL, Priesterbach LP, Uiterwaal CS, et al. Internet-based therapy for adolescents with chronic fatigue syndrome: long-term follow-up. Pediatrics 2013;131:e1788-95.

23. Díaz-Caneja Greciano A, Rodríguez Sosa JT, Aguilera Albesa S, et al. [Chronic fatigue syndrome in a 15-year-old girl]. An Pediatr 2007;67:74-7.

24. Chalder T, Tong J, Deary V. Family cognitive behaviour therapy for chronic fatigue syndrome: an uncontrolled study. Arch Dis Child 2002;86:95-7.

25. Lloyd S, Chalder T, Sallis HM, et al. Telephone-based guided self-help for adolescents with chronic fatigue syndrome: a nonrandomised cohort study. Behav Res Ther 2012;50:304-12.

26. van de Putte EM, Engelbert RH, Kuis W, et al. Alexithymia in adolescents with chronic fatigue syndrome. J Psychosom Res 2007:63:377-80

27. Zigmond AS, Snaith RP. The hospital anxiety and depression scale. Acta Psychiatr Scand 1983;67:361-70.

28. Papay JP, Spielberger CD. Assessment of anxiety and achievement in kindergarten and first- and second-grade children. J Abnorm Child Psychol 1986;14:279-86.

29. Spence SH. Structure of anxiety symptoms among children: a confirmatory factor-analytic study. J Abnorm Psychol 1997;106:280-97.

30. March JS, Parker JD, Sullivan K, et al. The Multidimensional Anxiety Scale for Children (MASC): factor structure, reliability, and validity. $J$ Am Acad Child Adolesc Psychiatry 1997;36:554-65.

31. Goodman R, Ford T, Richards H, et al. The Development and Wellbeing Assessment: description and initial validation of an integrated assessment of child and adolescent psychopathology. J Child Psychol Psychiatry 2000;41:645-55.

32. Nijhof SL, Maijer K, Bleijenberg G, et al. Adolescent chronic fatigue syndrome: prevalence, incidence, and morbidity. Pediatrics 2011;127:e1169-75.
33. Nijhof SL, Bleijenberg G, Uiterwaal CS, et al. Effectiveness of internet-based cognitive behavioural treatment for adolescents with chronic fatigue syndrome (FITNET): a randomised controlled trial. Lancet 2012;379:1412-8.

34. Rimes KA, Papadopoulos AS, Cleare AJ, et al. Cortisol output in adolescents with chronic fatigue syndrome: pilot study on the comparison with healthy adolescents and change after cognitive behavioural guided self-help treatment. J Psychosom Res 2014;77:409-14.

35. Creswell C, Waite P, Cooper PJ. Assessment and management of anxiety disorders in children and adolescents. Arch Dis Child 2014;99:674-8.

36. Crawley E, Collin SM, White PD, et al. Treatment outcome in adults with chronic fatigue syndrome: a prospective study in England based on the CFS/ME National Outcomes Database. QJM 2013;106:555-65.

37. Szigethy E, Kenney E, Carpenter J, et al. Cognitive-behavioral therapy for adolescents with inflammatory bowel disease and subsyndromal depression. J Am Acad Child Adolesc Psychiatry 2007;46:1290-8.

38. Ahmadi S, Tabibi Z, Ali M, et al. Effectiveness of group cognitivebehavioral therapy on anxiety, depression and glycemic control in children with type 1 diabetes. Int J Pediatr 2014;2:165-71.

39. Bennett S, Shafran R, Coughtrey A, et al. Psychological interventions for mental health disorders in children with chronic physical illness: a systematic review. Arch Dis Child 2015;100:308-16.

40. PRISMA. PRISMA flow diagram, 2009.

41. Sharpe MC, Archard LC, Banatvala JE, et al. A report--chronic fatigue syndrome: guidelines for research. $J$ R Soc Med $1991 ; 84: 118-21$. 